\title{
Implementação e Avaliação da Eficiência de um Algorítmo MPPT P\&O Baseado em Corrente para Geração Fotovoltaica
}

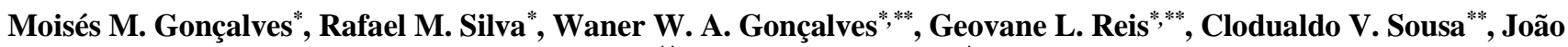 \\ L. Silva**, Seleme I. Seleme Jr." \\ "Programa de pós Graduação em engenharia elétrica Universidade Federal de Minas Gerais (UFMG), \\ Av. Antônio Carlos 6627, 31270-901, Belo Horizonte, MG, Brasil \\ ** Departamento de Engenharia Elétrica, Universidade Federal de Itajubá (UNIFEI), \\ Av. Irmã Ivone Drumond, 200, 35903-087, Itabira, MG, Brazil \\ e-mail:moisesgonc@unifei.edu.br, rafaellmario@ufmg.br@ \\ waner@unifei.edu.br@, geovanereis@unifei.edu.br, clodualdosousa@unifei.edu.br, \\ joaolucas.silva@unifei.edu.br, seleme@cpdee.ufmg.br $\mathbb{D}$.
}

\begin{abstract}
In recent years there has been a significant increasing of distributed generation integration to the electrical mains, where it is possible to highlight photovoltaic systems. One of the key points in the implementation of these systems is the elaboration and tuning of the maximum power tracking (MPPT) algorithm. The present paper evaluates the impacts of the MPPT algorithm tuning, such as the choice of the increment/decrement step and the update time of the tracking variables, over the efficiency of the energy extraction process. For this, a $\mathrm{P} \& \mathrm{O}$ (disturb and observe) algorithm using the photovoltaic panels current as a control variable is implemented in a $5.1(\mathrm{~kW})$ system.
\end{abstract}

Resumo: Nos últimos anos houve um significativo aumento na integração dos sistemas de geração distribuída a rede elétrica, onde pode-se destacar os sistemas fotovoltaicos. Um dos pontos primordiais na implementação destes sistemas é a elaboração e sintonia do algoritmo de rastreamento de máxima potência (MPPT). O presente trabalho avalia os impactos da sintonia do algoritmo MPPT, como a escolha do passo e do tempo de atualização das variáveis de rastreamento, sobre a eficiência do processo de extração de energia. Para isso, um algoritmo $\mathrm{P} \& \mathrm{O}$ (perturbe e observe) utilizando a corrente como variável de controle é implementado em um sistema de $5,1(\mathrm{~kW})$.

Keywords: Efficiency calculation, PV systems control, distributed generation, max power point tracking (MPPT), photovoltaic systems.

Palavras-chaves: Calculo de Eficiência, Controle de sistemas PV, Geração distribuída, max power point tracking (MPPT), sistemas fotovoltaicos.

\section{INTRODUÇÃO}

A geração distribuída (GD) pode ser definida como a geração de energia elétrica próxima ao consumidor. Segundo dados da ANEEL (ANEEL, 2018), a fonte mais empregada para a implantação de sistemas de geração distribuída no Brasil é a fotovoltaica, do inglês Photovoltaic (PV). Sua elevada taxa de empregabilidade nos dias atuais está associado a alguns fatores como a redução dos custos dos módulos PV, a regulamentação dos micro e mini geradores distribuídos através da resolução REN ANEEL 482/2012 (ANEEL, 2012) e 687/2015 (ANEEL, 2015) e principalmente devido a disponibilidade do recurso, uma vez que, o Brasil possui um grande potencial para geração de energia fotovoltaica ao longo de todo o seu território.

Uma célula PV, consiste da junção de materiais semicondutores que transformam a radiação solar em energia elétrica através do efeito fotovoltaico (Silva et al., 2017a). Elas apresentam uma característica não linear entre a tensão e corrente, por consequência, com a potência. Além disso, as características dos módulos PV são fortemente influenciados pelas condições ambientais, como temperatura e irradiação solar no qual os mesmos são submetidos (Mukund R. Patel, 2005). Desta forma, um fator fundamental para o aumento do aproveitamento da energia gerada pelos módulos PV é a capacidade da extração da máxima potência, do inglês Max Power Point Tracking (MPPT), para diferentes condições de temperatura e irradiância solar.

Diversos algoritmos de MPPT são apresentados na literatura, sendo os mais difundidos atualmente o Perturbe e Observe (Pertubation \& Observation - P\&O) (Midya et al., 1996) e Condutância Incremental (Incremental Conductance IncCond) (Hussein et al., 1995). Um dos pontos básicos para o desenvolvimento do algoritmo MPPT é a definição do passo ou ganho $\left(k_{m p p t}\right)$ de rastreamento e do tempo de atualização $(\Delta t)$ das variáveis (Schmidt et al., 2009; Subudhi and Pradhan, 2013). Entretanto, poucos trabalhos relacionam a escolha destas grandezas com a eficiência do rastreamento, tanto em regime permanente quanto em condições transitórias.

A maioria dos trabalhos tem como foco a implementação de algoritmos que utilizam a tensão como variável de rastreamento, uma vez que esta apresenta uma característica mais bem comportada com a variação das condições ambientais em comparação com a corrente dos módulos (Bellinaso et al., 2019). No entanto, a escolha da corrente é mais intuitiva considerando as variações de demanda da carga, como discutido em (Aureliano Gomes de Brito et al., 2012; de Brito et al., 2011), onde são apresentados diversos algoritmos de MPPT utilizando a corrente como variável de controle. 
Em sistemas que utilizam estágios c.c.-c.c. intermediários entre a fonte primária e o barramento c.c. do conversor conectado à rede (Jesus et al., 2016; Kamdi and Joshi, 2017), é necessário inserir uma malha de controle da tensão do arranjo de painéis externa a malha de controle de corrente do conversor c.c-c.c., o que aumenta a complexidade, custo computacional e tempo de resposta. Embora, o controle em cascata da tensão e corrente possa contribuir em uma maior robustez para o sistema.

O objetivo deste trabalho é discutir os efeitos do ganho de rastreamento e do tempo de atualização sobre a eficiência de um algoritmo $\mathrm{P} \& \mathrm{O}$ que utiliza a corrente como variável de controle, onde esta avaliação será feita através de simulações computacionais e resultados experimentais em uma bancada de $5.1(\mathrm{~kW})$.

O presente artigo é organizado da seguinte forma: A Seção 2 discute os efeitos das condições ambientais sobre as características dos painéis fotovoltaicos. Na Seção 3 é apresentada a modelagem e a descrição do sistema de controle, a Seção 4 descreve o algoritmo de MPPT e apresenta as figuras de mérito para análise da eficiência do algoritmo. Na seção 5 são apresentados os resultados obtidos através de simulação computacional e na Seção 6 os resultados experimentais. Por fim, as conclusões são realizadas na Seção 7.

\section{CARACTERÍSTICAS DOS PAINÉIS}

A Fig. 1 mostra curvas características típicas da corrente em função da tensão (Fig. 1(a)), potência em função da tensão (Fig. 1(b)) e potência em função da corrente (Fig. 1(c)) de um módulo fotovoltaico para diferentes condições de irradiância solar $(G)$ e temperatura $(T)$. A corrente de curto circuito $\left(I_{s c}\right)$, medida com os terminais do módulo PV em curto, é reduzida proporcionalmente com a irradiância, já a tensão de circuito aberto $\left(V_{o c}\right)$, medida com os terminais do módulo $\mathrm{PV}$ em aberto, é diretamente afetada pela temperatura, sendo que, para cada condição haverá um valor máxima potência que poderá ser extraído.

Considere as curvas para a condição padrão de irradiância e temperatura, i.e., $G=1000(\mathrm{~W} / \mathrm{m} 2)$ e $T=25\left({ }^{\circ} \mathrm{C}\right)$ (curva preta). Entre a região de circuito aberto do módulo e a máxima potência (região vermelha), variações pequenas de tensão irão proporcionar grandes alterações na corrente do módulo. Já entre a região de curto circuito e a máxima potência (região azul), variações mínimas de corrente irão produzir grandes alterações no valor de tensão do módulo PV, sendo muito mais restritivo do que a alteração de tensão no plano azul.

\section{Sistemas Fotovoltaicos}

O diagrama simplificado do sistema de conversão de energia é mostrado na Fig. 2. O MSC (Mains Side Converter) é responsável por entregar a energia c.c. gerada pelo sistema fotovoltaico a rede elétrica c.a., controlando a tensão do barramento c.c. com valor fixo $V_{d c}$. O conversor boost é responsável por garantir a extração da máxima potência dos módulos PV. O boost opera com tensão de saída fixa, já que o barramento c.c. é controlado pelo conversor MSC. Entre o arranjo e o boost é utilizado o capacitor $C_{b}$ para redução das oscilações instantâneas de potência nos módulos, amortizando os impactos do chaveamento na eficiência da conversão de energia (Kjaer et al., 2005).

\subsection{Modelagem do conversor boost}

O circuito equivalente do ponto de vista do controle do conversor Boost se encontra na Fig. 3, onde $i_{p h}$ é a corrente de efeito fotovoltaico, $r_{p v}$ é a resistência dinâmica equivalente e $i_{p v}$ a corrente de saída do arranjo fotovoltaico, $i_{C b}$ a corrente do capacitor de entrada, $i_{l}$ a corrente, $L_{b}$ a indutância e $r_{b}$ a resistência do indutor do conversor Boost.

(a)

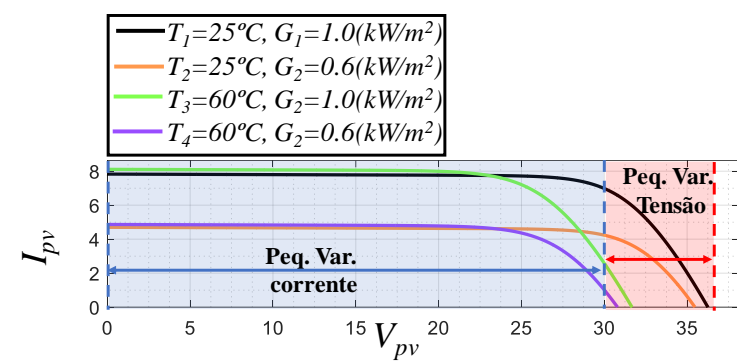

(b)

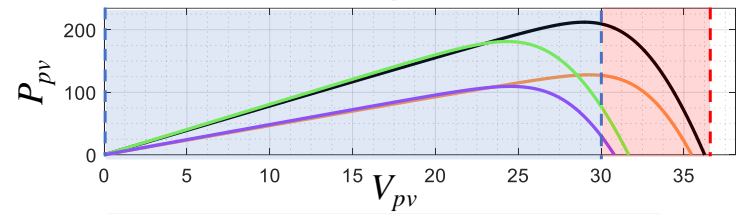

(c)

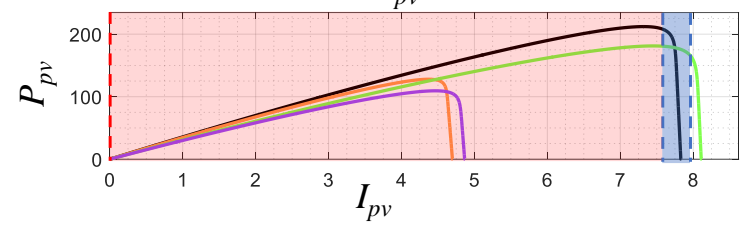

Fig. 1: Curvas características típicas de um módulo fotovoltaico, (a) tensão $\mathrm{x}$ corrente, (b) potência x tensão e (c) potência x corrente.

Array de

paineis

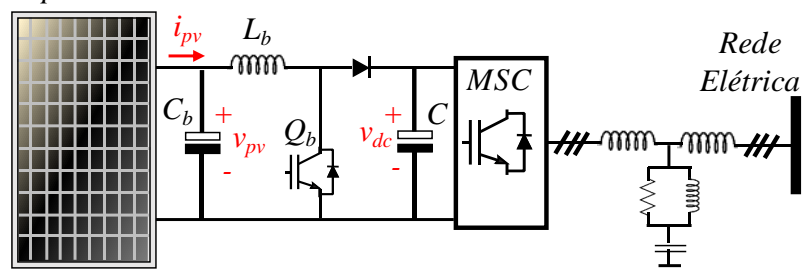

Fig. 2: Conversor de energia fotovoltaica conectado a rede elétrica

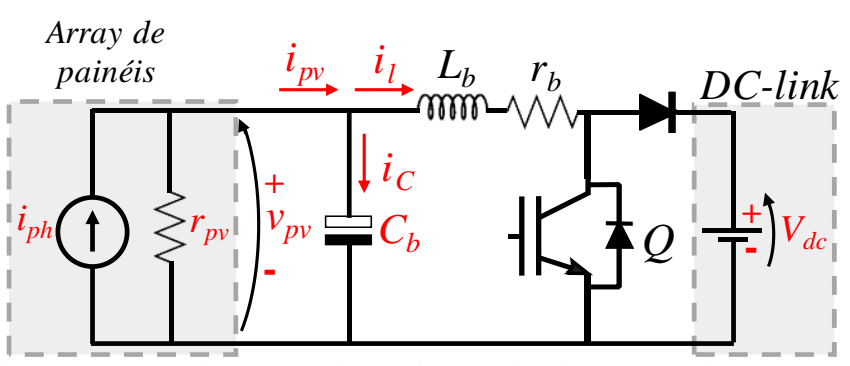

Fig. 3: Modelo equivalente do sistema fotovoltaico do ponto de vista do controle do conversor boost.

Aplicando as leis de Kirchoff ao circuito da Fig. 3, considerando apenas a operação na condição de condução continua, as relações (1) e (2) são obtidas (Urtasun et al., 2013).

$$
\begin{gathered}
i_{p v}=i_{C b}+i_{l}=C_{b} \cdot \frac{d v_{p v}}{d t}+i_{l} \\
v_{p v}=L_{b} \cdot \frac{d i_{l}}{d t}+r_{l} \cdot i_{l}+(1-d) \cdot V_{d c}
\end{gathered}
$$

onde $d$ é a razão cíclica do conversor. Assumindo que a dinâmica do painel PV é mais lenta que a dinâmica do 
conversor Boost, as correntes provenientes do painel podem ser tratadas como distúrbios ao controle, ou seja:

$$
C_{b} \cdot \frac{d v_{p v}}{d t}+i_{l}=0
$$

Substituindo (2) em (3), aplicando a transformada de Laplace e rearranjando os termos é obtida a função de transferência que relaciona a razão cíclica d com a corrente no indutor $i l$, como segue:

$$
G_{p}(s)=\frac{I_{l}(s)}{(1-d)(s)}=\frac{V_{d c} \cdot C_{b} \cdot s}{C_{b} \cdot L \cdot s^{2}+C_{b} \cdot r_{l} \cdot s+1}
$$

\subsection{Projeto do controlador de corrente}

Assumindo que o valor da capacitância de entrada $C b$ é elevada o suficiente, de forma que não haverá uma descarga significativa do capacitor, a seguinte aproximação pode ser realizada (Puukko et al., 2011; Urtasun et al., 2013):

$$
i_{l} \approx i_{p v}
$$

ou seja, a corrente de saída do módulo é aproximadamente igual a corrente de entrada do conversor boost. Logo, o algoritmo de MPPT irá gerar diretamente a referência para a malha de controle da corrente no indutor, como é mostrado na Fig. 4, onde $G p(\mathrm{~s})$ é a função de transferência do conversor boost, e $C(\mathrm{~s})$ a função o controlador.

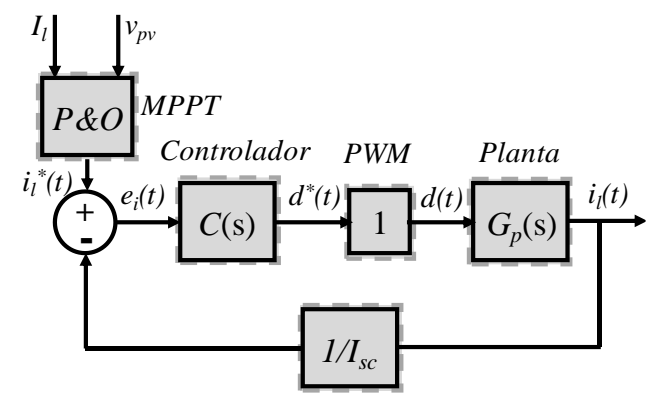

Fig. 4: Diagrama de blocos de controle do conversor boost.

Para a implementação do sistema, é utilizado um controlador do tipo proporcional-integral (PI), por apresentar fácil implementação e ser bem-comportado com relação a sobressinal (overshoot) e tempo de acomodação. Para a sintonia deste controlador foi utilizado o critério de resposta em frequência baseado na metodologia desenvolvida em (Buso and Mattavelli, 2015; Phillips and Harbor, 1996). Como projeto dos controladores foram estipulados os seguintes critérios, frequência de cruzamento de ganho igual a um décimo da frequência de chaveamento do boost, possibilitando desconsiderar a dinâmica do conversor e margem de fase igual a $60^{\circ}$, de forma a se ter a melhor relação possível entre tempo de resposta e máximo sobressinal (overshoot).

Os parâmetros do conversor são apresentados na Tabela 1, onde os ensaios para a obtenção dos mesmos são descritos em (Silva et al., 2017b). A resposta em frequência do sistema em malha aberta sem e com o controlador é mostrado na Fig. 5, onde foram obtidos: ganho proporcional $k p=0,2887(1 / \mathrm{A}) \mathrm{e}$ ganho integral $k i=1057,0(1 / \mathrm{A})$.

\section{ALGORITMO DE RASTREAMENTO DE MÁXIMA DE POTÊNCIA BASEADO EM CORRENTE}

Neste trabalho é utilizado um algoritmo de rastreamento $\mathrm{P} \& \mathrm{O}$ que emprega como variável de rastreamento a corrente do arranjo. Como o próprio nome sugere, o algoritmo $\mathrm{P} \& \mathrm{O}$ consiste em perturbar a variável de rastreamento em um determinado sentido e observar o comportamento da potência de saída. Logo, o algoritmo $\mathrm{P} \& \mathrm{O}$ apresenta um erro em regime estacionário intrínseco a sua implementação, uma vez que, o intuito é sempre perturbar a variável de rastreamento em torno do ponto de máxima potência. Quanto menor for o passo $k_{m p p t}$,

\begin{tabular}{|c|c|c|c|}
\hline & Parâmetros & Símbolo & Valor \\
\hline \multirow{2}{*}{ Boost } & Indutância & $L b$ & $2,71(\mathrm{mH})$ \\
\hline & Resistência & $R b$ & $71(\mathrm{~m} \Omega)$ \\
\hline \multicolumn{2}{|c|}{ Capacitor de Entrada } & $C p v$ & $470(\mu \mathrm{F})$ \\
\hline \multicolumn{2}{|c|}{ Tensão Saída } & $V d c$ & $450(\mathrm{~V})$ \\
\hline \multicolumn{2}{|c|}{ Freq. Chaveamento } & $f s w$ & $10,08(\mathrm{kHz})$ \\
\hline
\end{tabular}
menor será o erro, no entanto mais lento será o rastreamento da potência.

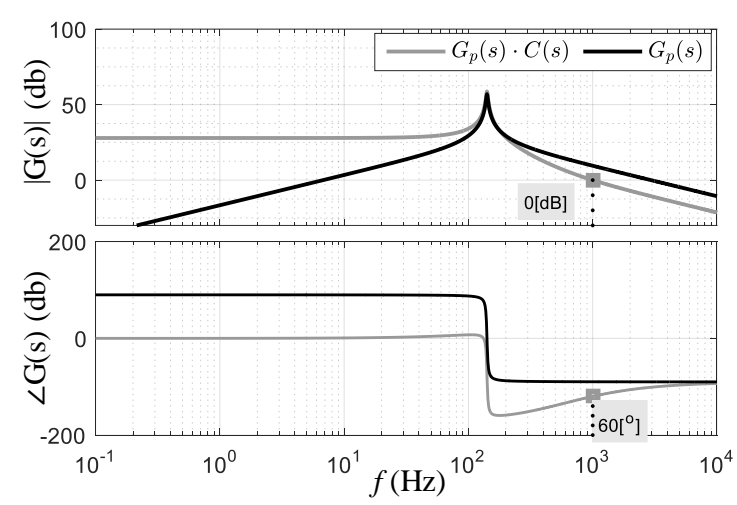

Fig. 5: Reposta em frequência do sistema em malha aberta sem e com controlador PI.

Outro parâmetro importante é a frequência de atualização do algoritmo $(\Delta t)$, quanto mais rápida a atualização, mais rápido o algoritmo encontrará o ponto de máximo, no entanto, oscilações muito rápidas refletem em grandes variações no valor de potência, além disso, podem levar o controle de corrente a instabilidade. Desta forma, a escolha de $k_{m p p t} \mathrm{e} \Delta t$ têm grande influência na eficiência do processo de rastreamento de máxima potência. A Fig. 6 mostra o fluxograma do algoritmo $\mathrm{P} \& \mathrm{O}$.

\subsection{Definição da eficiência do MPPT}

Devido ao comportamento estocástico das condições ambientais, uma correta avaliação da eficiência do MPPT deve levar em conta os seguintes aspectos (Jesus et al., 2016):

- Para condições ambientais diversas em regime permanente será medida a Eficiência Estática (nest) (Schärf and Häberlin, 2009);

- Durante as transições entre as condições ambientais, será avaliada a Eficiência Dinâmica ( $\eta$ din) (Schärf and Häberlin, 2009).

A eficiência de rastreamento do Algoritmo MPPT ( $\left.\eta_{M P P T}\right)$ pode ser calculada por (Schärf and Häberlin, 2009): 


$$
\eta_{M P P T}=\int_{0}^{T_{M}} \frac{v_{P V}(t) \cdot i_{P V}(t)}{P_{M P P}(t)} \cdot d t
$$

onde, $v p v$ e ipv são a tensão e corrente do arranjo fotovoltaico na entrada do conversor Boost, respectivamente, $P_{M P P}$ a máxima potência disponível do arranjo fotovoltaico e $T_{M} \mathrm{o}$ tempo de medição.

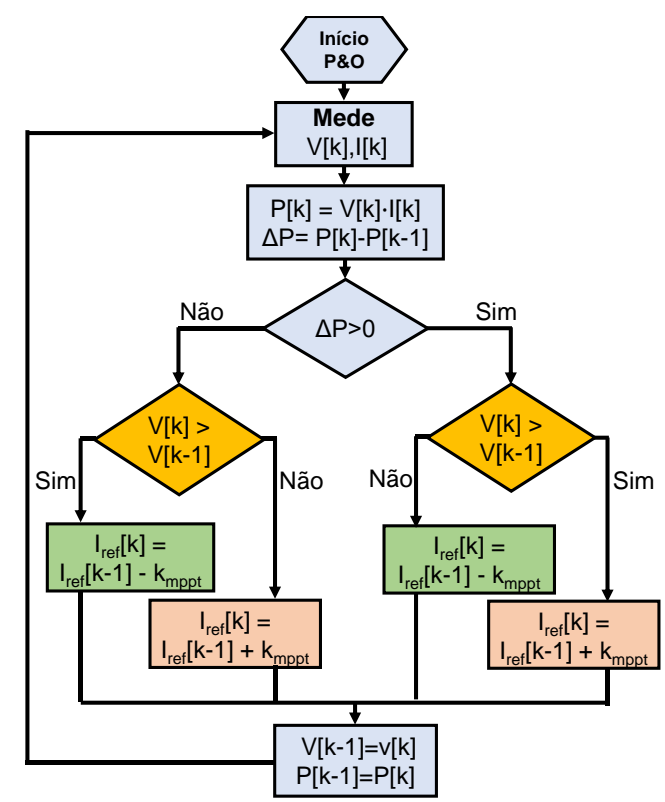

Fig. 6: Fluxograma do algoritmo $\mathrm{P} \& \mathrm{O}$ para o rastreamento de máxima potência utilizando a corrente

\section{SIMULAÇÃO DO CONVERSOR BOOST}

Para avaliação do comportamento dinâmico do conversor boost, foi realizada uma simulação computacional através do software PLECS ${ }^{\circledR}$, utilizando os parâmetros descritos na Tabela 1 Os ganhos do controlador foram discretizados utilizando a transformação Bilinear (Tustin) (Phillips and Harbor, 1996) com frequência de amostragem igual ao dobro da frequência de chaveamento. A Fig. 7 mostra a resposta do sistema de controle para um sinal do tipo degrau de amplitude de 8,5(A), onde são obtidos um máximo sobressinal de $24(\%)$ e um tempo de acomodação de 3,5(ms).

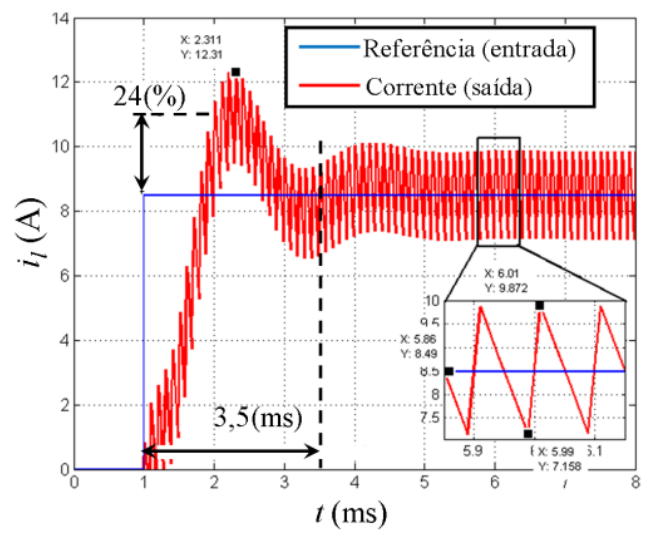

Fig. 7: Resposta ao degrau do controle do conversor boost. (resultado de simulação)

O valor elevado de sobressinal pode ser explicado através da função de transferência do sistema na equação (4), que apresenta um zero na origem do plano complexo. Essa característica (zeros lentos) implica em valores mais significativos de sobressinal (overshoot), além disso, por estar na origem há uma tendência de anular o efeito do controlador PI, o que explica o elevado valor do ganho integrativo calculado na seção anterior.

\section{RESULTADOS EXPERIMENTAIS}

A bancada experimental onde foi realizada a implementação e validação do algoritmo MPPT proposto e do controle trata-se de um sistema de geração hibrido, onde maiores descrições podem ser encontrada em (Silva et al., $2017 b, 2017$ c). Para maior flexibilização dos ensaios foi utilizada a fonte CC modelo XR400-20 para emular um arranjo fotovoltaico de $5,1 \mathrm{~kW}$ utilizando os dados dos painéis YL255P-29b, com $I s c=17,76$ A e Voc $=370 \mathrm{~V}$.

\subsection{Resposta dinâmica do conversor boost}

Inicialmente, foi avaliada a resposta dinâmica do conversor boost para um sinal do tipo degrau com amplitude de $4 \mathrm{~A}$, com a tensão de entrada mantida fixa pela fonte em $370 \mathrm{~V}$ e tensão de saída (barramento c.c.) de 450 V. Observa-se na Fig. 8 que o máximo sobressinal é de $25 \%$ e tempo de acomodação 3,5 ms.

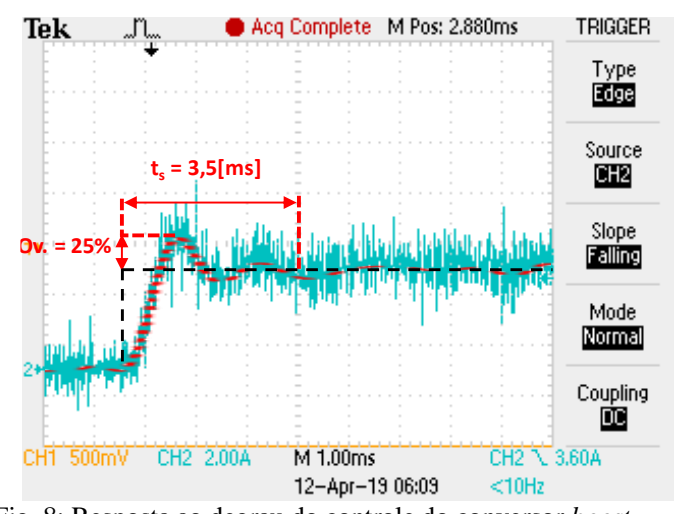

Fig. 8: Resposta ao degrau do controle do conversor boost. (resultado experimental)

\subsection{Determinação dos parâmetros do MPPT}

Um dos pontos mais importantes na implementação do algoritmo MPPT é a determinação do passo (kmppt) de rastreamento e do tempo de atualização $(\Delta t)$ das variáveis, pois estes influem diretamente sobre a eficiência da conversão de energia. Para o presente trabalho a determinação desses valores foi realizada com base na eficiência estática, onde foi definido range no qual este parâmetro seria avaliado, da seguinte forma:

- Tempo mínimo de atualização das variáveis é de $10 \mathrm{~ms}$, i.e., dez vezes mais lento que a malha de controle de corrente, para que o MPPT não tenha grande influência sobre a dinâmica da malha de controle. Tempo máximo de atualização das variáveis de $110 \mathrm{~ms}$, ou seja, aproximadamente cem vezes mais lento que a malha de corrente, de forma que não se tenha um tempo de resposta muito elevado;

- Valor mínimo do passo de rastreamento de 0,05 A $(0,3 \%$ de $I s c)$ para que não se tenha um tempo de resposta muito elevado. Valor máximo do passo de rastreamento de $0,25 \mathrm{~A}(1,0 \%$ de $I s c)$ para que não se tenha grande perturbações na dinâmica de controle.

Foram realizados diversos ensaios variando kmppt e $\Delta t$ dentro do intervalo descrito anteriormente, nas condições 
padrão de teste $\left(G=1000 \mathrm{~W} / \mathrm{m} 2\right.$ e $\left.T=25{ }^{\circ} \mathrm{C}\right)$, obtendo a superfície mostrada na Fig. 9, onde são destacados os dois pontos de maior eficiência. Para uma melhor avaliação dos pontos, também se faz necessário avaliar o comportamento destes ganhos para diferentes condições ambientais através da eficiência dinâmica.

Logo, os pontos em destaque na Fig. 9 serão avaliados em diferentes condições ambientais e durante a variação das mesmas através da eficiência dinâmica. Inicialmente foi realizada uma variação em rampa de irradiância de $360 \mathrm{~W} / \mathrm{m}^{2}$ a $1000\left(\mathrm{w} / \mathrm{m}^{2}\right)$ com $T=25^{\circ} \mathrm{C}$, como mostrado na Fig. 10. Posteriormente, foi realizada uma variação em rampa no valor de temperatura de $25^{\circ} \mathrm{C}$ à $80^{\circ} \mathrm{C} \operatorname{com} G=1000 \mathrm{~W} / \mathrm{m}^{2}$, como se observa na Fig. 11.

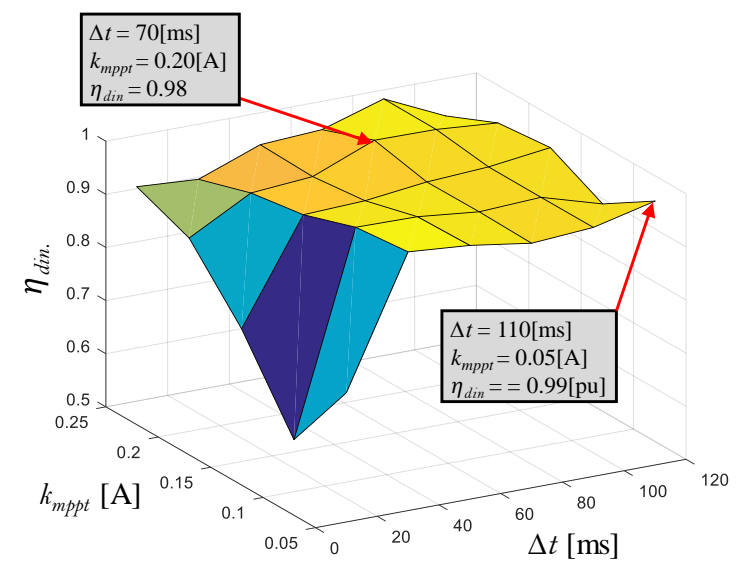

Fig. 9: Eficiência dinâmica para diferentes valores de $k_{m p p t} \mathrm{e} \Delta t$. (resultado experimental)

Os valores de eficiência calculadas para os testes de elevação e redução da irradiância e temperatura são descritos na Fig. 10 e Fig. 11, respectivamente. As duas situações apresentaram elevados valores de eficiência, entretanto, comportamentos dinâmicos bem distintos. Para a primeira condição, por operar com maior frequência de atualização e passo de incremento, observa-se grandes variações instantâneas no valor da potência medida.

Já para o segundo conjunto de parâmetros, a medição de potência possui um comportamento dinâmico melhor em comparação ao primeiro, principalmente nas condições ambientais estáticas. Nota-se que para a rampa decrescente de irradiação, a eficiência dinâmica apresenta uma redução significativa em relação à crescente, pois, a variação da irradiação sobre o módulo tem grande influência sobre o valor da corrente de máxima potência. Logo, quando da redução no valor de $G$, a corrente de máxima potência da condição anterior apresenta valor próximo a corrente de curto para o novo estado de operação e o controle de corrente tende a curto-circuitar a saída do módulo, levando-o a saturação.

Tabela 2: Eficiência - Variações das Condições Ambientais

\begin{tabular}{|c|c|c|c|c|}
\hline \multirow{2}{*}{ Conjunto } & \multirow{2}{*}{ Variação } & \multirow{2}{*}{$\begin{array}{l}\text { Eficiência } \\
\text { Estática } \\
\left(\eta_{\text {est }}\right)(\%)\end{array}$} & \multicolumn{2}{|c|}{$\begin{array}{l}\text { Eficiência Dinâmica } \\
\left(\eta_{\operatorname{din}}\right)(\%)\end{array}$} \\
\hline & & & $\begin{array}{l}\text { Rampa } \\
\text { Crescente }\end{array}$ & $\begin{array}{l}\text { Rampa } \\
\text { Decrescente }\end{array}$ \\
\hline \multirow{2}{*}{1} & Irradiância & 99,68 & 97,63 & 95,69 \\
\hline & Temperatura & 99,68 & 99,40 & 99,56 \\
\hline \multirow{2}{*}{2} & Irradiância & 94,95 & 93,05 & 92,24 \\
\hline & Temperatura & 94,95 & 95,08 & 92,48 \\
\hline
\end{tabular}

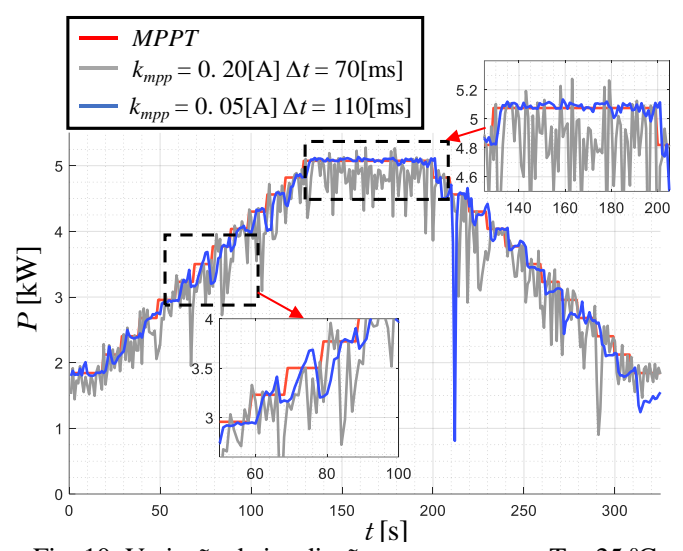

Fig. 10: Variação da irradiação em rampa com $\mathrm{T}=25^{\circ} \mathrm{C}$. (resultado experimental)

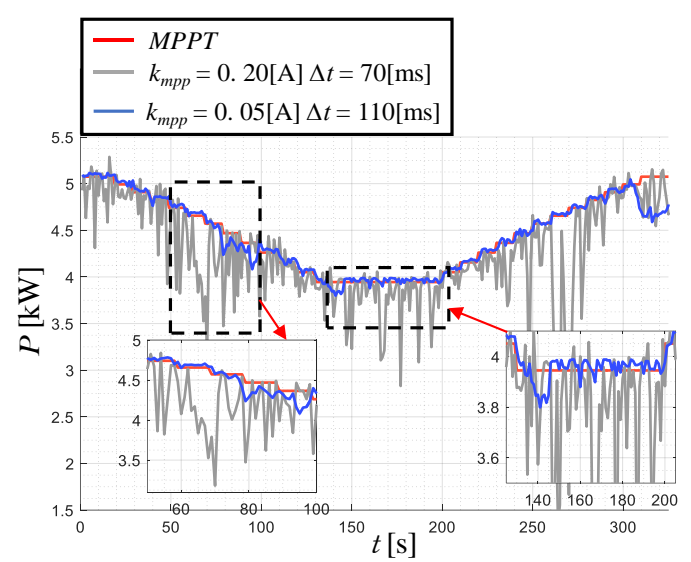

Fig. 11: Variação da temperatura em rampa com $\mathrm{G}=1 \mathrm{~kW} / \mathrm{m}^{2}$. (resultado experimental)

\section{CONCLUSÕES}

Este trabalho teve como objetivo investigar os efeitos dos parâmetros do algoritmo MPPT, como ganho de rastreamento e tempo de atualização, sobre a eficiência no processo de extração de energia utilizando um algoritmo de MPPT P\&O baseado na variação da corrente.

A principal vantagem do algoritmo de MPPT baseado em corrente é a eliminação da malha de controle externa da tensão do arranjo PV, por gerar o valor da referência de corrente do boost de forma direta. Reduzindo o tempo de resposta e o esforço computacional. Além disso, há uma maior flexibilidade na operação do sistema, pois, quando necessário é possível controlar a tensão de saída do boost e o rastreamento de máxima potência de forma simultânea. Embora seja um benefício, a ausência da malha para o controle da tensão dos painéis contribui para a redução do amortecimento nos efeitos da presença do zero na origem do plano complexo, efeitos estes que podem ser compensados através de uma sintonia mais precisa dos controladores. A escolha dos parâmetros do algoritmo MPPT tem grande influência sobre a eficiência no processo de conversão de energia, a influência de $G$ sobre a corrente de MPP pode forçar o deslocamento do ponto de operação para a região de curto-circuito do arranjo, o que pode ser mitigado através da correta seleção dos parâmetros do algoritmo de MPPT.

\section{AGRADECIMENTOS}

O presente trabalho foi realizado com o apoio financeiro da CAPES - Brasil. 


\section{REFERÊNCIAS}

ANEEL, A.N. de E.E., 2018. Technical Information.

ANEEL, A.N. de E.E., 2015. Resolução Normativa no 687, de 24 de Novembro de 2015.

ANEEL, A.N. de E.E., 2012. Resolução Normativa no 482, de 17 de abril de 2012.

Aureliano Gomes de Brito, M., Poltronieri Sampaio, L., de Azevedo e Melo, G., Alberto Canesin, C., 2012. Contribution For The Study Of The Main Pv Maximum Power Point Tracking Methods. Eletrônica Potência 17, 592-600. https://doi.org/10.18618/REP.2012.3.592600

Bellinaso, L.V., Figueira, H.H., Basquera, M.F., Vieira, R.P., Gründling, H.A., Michels, L., 2019. Cascade Control With Adaptive Voltage Controller Applied to Photovoltaic Boost Converters. IEEE Trans. Ind. Appl. 55, 1903-1912. https://doi.org/10.1109/TIA.2018.2884904

Buso, S., Mattavelli, P., 2015. Digital control in power electronics, 2nd ed. Morgan\&Calypool.

de Brito, M.A.G., Sampaio, L.P., Luigi, G., e Melo, G.A., Canesin, C.A., 2011. Comparative analysis of MPPT techniques for PV applications, in: 2011 International Conference on Clean Electrical Power (ICCEP). Presented at the 2011 International Conference on Clean Electrical Power (ICCEP), IEEE, Ischia, Italy, pp. 99-104. https://doi.org/10.1109/ICCEP.2011.6036361

Hussein, K.H., Muta, I., Hoshino, T., Osakada, M., 1995. Maximum photovoltaic power tracking: an algorithm for rapidly changing atmospheric conditions. Transm. Distrib. IEE Proc. - Gener. 142, 59-64. https://doi.org/10.1049/ipgtd: 19951577

Jesus, V.M.R. de, Xavier, L.S., Cupertino, A.F., Pereira, H.A., Mendes, V.F., 2016. Comparison of MPPT strategies applied in three-phase photovoltaic inverters during harmonic current compensation, in: 2016 12th IEEE International Conference on Industry Applications (INDUSCON). Presented at the 2016 12th IEEE International Conference on Industry Applications (INDUSCON), pp. $1-8$. https://doi.org/10.1109/INDUSCON.2016.7874591

Kamdi, N., Joshi, P.B., 2017. Simulation of three-phase twostage grid connected photovoltaic system using Cuk converter, in: 2017 Innovations in Power and Advanced Computing Technologies (i-PACT). Presented at the 2017 Innovations in Power and Advanced Computing Technologies (i-PACT), pp. 1-5. https://doi.org/10.1109/IPACT.2017.8245077

Kjaer, S.B., Pedersen, J.K., Blaabjerg, F., 2005. A review of single-phase grid-connected inverters for photovoltaic modules. IEEE Trans. Ind. Appl. 41, 1292-1306. https://doi.org/10.1109/TIA.2005.853371

Midya, P., Krein, P.T., Turnbull, R.J., Reppa, R., Kimball, J., 1996. Dynamic maximum power point tracker for photovoltaic applications, in: PESC Record. 27th Annual IEEE Power Electronics Specialists Conference. Presented at the PESC Record. 27th Annual IEEE Power Electronics Specialists Conference, pp. 1710-1716 vol.2. https://doi.org/10.1109/PESC.1996.548811

Mukund R. Patel, 2005. Wind and Solar Power Systems: Design, Analysis, and Operation, 2nd ed. CRC Press.
Phillips, C.L., Harbor, R.D., 1996. Feedback Control System, 3rd ed. Prentice Hall.

Puukko, J., Nousiainen, L., Suntio, T., 2011. Effect of minimizing input capacitance in VSI-based renewable energy source converters, in: 2011 IEEE 33rd International Telecommunications Energy Conference (IN ${ }^{\mathrm{TEL}} \mathrm{EC}$ ). Presented at the 2011 IEEE 33rd International Telecommunications Energy Conference (IN $\left.{ }^{\mathrm{TEL}} \mathrm{EC}\right)$, pp. 19. https://doi.org/10.1109/INTLEC.2011.6099891

Schärf, P., Häberlin, H., 2009. New Procedure for Measuring Dynamic MPP-Tracking Efficiency at Grid-Connected PV Inverters. 24th Eur. Photovolt. Sol. Energy Conf. 21-25 Sept. 2009 Hambg. Ger. 3631-3637. https://doi.org/10.4229/24thEUPVSEC2009-4BV.1.9

Schmidt, H., Burger, B., Bussemas, U., Elies, S., 2009. How fast does an MPP tracker really need to be?, in: 24th European Photovoltaic Solar Energy Conference. Presented at the European Photovoltaic Solar Energy Conference, Hamburg, https://doi.org/10.4229/24thEUPVSEC2009-4CO.8.3

Silva, J.L.D., Reis, G.L.D., Silva, R.M., Seleme, S.I., Meynard, T.A., Llor, A.M., 2017a. Design, modeling and identification of the Generation Side Converter in an 11.7 $\mathrm{kW}$ wind/photovoltaic hybrid renewable generation system, in: 2017 IEEE 8th International Symposium on Power Electronics for Distributed Generation Systems (PEDG). Presented at the 2017 IEEE 8th International Symposium on Power Electronics for Distributed Generation Systems (PEDG), pp. 1-8. https://doi.org/10.1109/PEDG.2017.7972548

Silva, J.L.D., Reis, G.L.D., Silva, R.M., Seleme, S.I., Meynard, T.A., Llor, A.M., 2017b. Design, modeling and identification of the Generation Side Converter in an 11.7 $\mathrm{kW}$ wind/photovoltaic hybrid renewable generation system, in: 2017 IEEE 8th International Symposium on Power Electronics for Distributed Generation Systems (PEDG). Presented at the 2017 IEEE 8th International Symposium on Power Electronics for Distributed Generation Systems (PEDG), pp. https://doi.org/10.1109/PEDG.2017.7972548

Silva, J.L.D., Reis, G.L.D., Silva, R.M., Seleme, S.I., Meynard, T.A., Llor, A.M., 2017c. Design, modeling and identification of the Mains Side Converter in an $11.7 \mathrm{~kW}$ wind/photovoltaic hybrid renewable generation system, in: 2017 IEEE 8th International Symposium on Power Electronics for Distributed Generation Systems (PEDG). Presented at the 2017 IEEE 8th International Symposium on Power Electronics for Distributed Generation Systems (PEDG), pp. 1-8. https://doi.org/10.1109/PEDG.2017.7972551

Subudhi, B., Pradhan, R., 2013. A Comparative Study on Maximum Power Point Tracking Techniques for Photovoltaic Power Systems. IEEE Trans. Sustain. Energy 4, 89-98. https://doi.org/10.1109/TSTE.2012.2202294

Urtasun, A., Sanchis, P., Marroyo, L., 2013. Adaptive Voltage Control of the DC/DC Boost Stage in PV Converters With Small Input Capacitor. IEEE Trans. Power Electron. 28, 5038-5048. https://doi.org/10.1109/TPEL.2013.2240702 